\title{
Quark and gluon spin-2 form factors to two-loops in QCD
}

\author{
Daniel de Florian, ${ }^{a}$ Maguni Mahakhud, ${ }^{b}$ Prakash Mathews, ${ }^{c}$ Javier Mazzitelli ${ }^{a}$ and \\ V. Ravindran ${ }^{d}$ \\ ${ }^{a}$ Departamento de Física, FCEyN, Universidad de Buenos Aires, \\ (1428) Pabellón 1, Ciudad Universitaria, Capital Federal, Argentina \\ ${ }^{b}$ Regional Centre for Accelerator-based Particle Physics, Harish-Chandra Research Institute, \\ Chhatnag Road, Jhunsi, Allahabad 211 019, India \\ ${ }^{c}$ Saha Institute of Nuclear Physics, \\ 1/AF Bidhan Nagar, Kolkata 700 064, India \\ ${ }^{d}$ The Institute of Mathematical Sciences, \\ C.I.T Campus, 4th Cross St, Tharamani Chennai, Tamil Nadu 600 113, India \\ E-mail: deflo@df.uba.ar, maguni@hri.res.in, \\ prakash.mathews@saha.ac.in, jmazzi@df.uba.ar, ravindra@imsc.res.in
}

ABSTRACT: We present complete two-loop radiative corrections to the graviton-quarkantiquark form factor $G^{*} \rightarrow q \bar{q}$ and graviton-gluon-gluon form factor $G^{*} \rightarrow g g$ in $\mathrm{SU}(N)$ gauge theory with $n_{f}$ light flavours using $d$-dimensional regularisation to all orders in $\varepsilon=d-4$. This is an important ingredient to next-to-next-to-leading order QCD corrections to hadronic scattering processes in models with large extra-dimensions where Kaluza-Klein graviton modes couple to Standard Model fields. We show that these form factors obey Sudakov integro-differential equation and the resulting cusp, collinear and soft anomalous dimensions coincide with those of electroweak vector boson and gluon form factors. We also find the universal behaviour of the infrared singularities in accordance with the proposal by Catani.

Keywords: NLO Computations, Phenomenology of Large extra dimensions

ARXIV EPRINT: 1312.6528 


\section{Contents}

1 Introduction 1

2 Two loop form factors $\quad 2$

$\begin{array}{llr}3 & \text { Infrared divergence structure } & 6\end{array}$

4 Conclusions $\quad 9$

$\begin{array}{ll}\text { A } \varepsilon \text { expansion of the two-loop form factors } & 10\end{array}$

\section{Introduction}

Extra dimension models are interesting scenarios to tackle the hierarchy problem. Depending on the geometry of the extra dimension(s), the two popular options are: (a) flat extra dimension model (ADD) [1-3] and (b) the warped extra dimension model (RS) with a large curvature [4]. In both these models, only the graviton is allowed to permeate the bulk which leads to different spectrum of spin-2 Kaluza-Klein (KK) modes in 4-dimensions. The spin-2, KK modes couple to the Standard Model (SM) particles through the energy momentum tensor of the SM.

These beyond SM model scenarios could alter the SM predictions by additional virtual KK mode exchanges and real KK mode productions. Dedicated groups in both ATLAS [5-7] and CMS $[8,9]$ collaborations are engaged in the analysis for extra dimension searches in various processes like di-lepton, di-photon, mono-jet, mono-photon productions etc. To put stringent bounds on the parameters of these BSM models, control on the theoretical uncertainties is essential. Renormalisation and factorisation scale dependences of a cross section to a particular order in perturbation theory give an estimate of the uncalculated higher order corrections. Presently next-to-leading order (NLO) QCD calculations have been done for di-lepton [10-12], di-photon $[13,14]$ and di-electroweak gauge boson [15-17] productions via virtual KK modes in addition to the SM contributions. These virtual contributions have been incorporated in the AMC@NLO frame work and results to $\mathrm{NLO}+\mathrm{PS}$ accuracy are now available for most of the di-final state processes $[18,19]$. In all these processes the factorisation scale dependence reduces substantially and in addition the NLO correction is in fact significant. For the above processes, the leading order (LO) is of the order $\mathcal{O}\left(\alpha_{s}^{0}\right)$, the renormalisation scale dependence starts only at NLO in QCD. To control the renormalisation scale dependence one would have to go to next-to-next-toleading order (NNLO) order.

A full NNLO QCD contribution requires the knowledge of graviton-quark-antiquark $G^{*} \rightarrow q \bar{q}$ and graviton-gluon-gluon $G^{*} \rightarrow g g$ form factors up to two-loop level in QCD 
in addition to double real emission and one-loop single real emission scatter processes at the parton level. Here $G^{*}$ denotes the virtual graviton. In this article we take the first step towards the full NNLO computation by evaluating these form factors to two-loop level in QCD by sandwiching the energy momentum tensor of the QCD part of the SM between on-shell gluon and quark states. We will also discuss the infrared (IR) structure of these form factors using Sudakov's integro-differential equation and Catani's proposal on two-loop QCD amplitudes.

In the next section, we will derive the two-loop form factors and in the section 3, we describe the infrared structure of these form factors. Finally we conclude in section 4 and the appendix contains the form factors expanded in powers of $\varepsilon$ to desired accuracy.

\section{Two loop form factors}

We work with the following action that describes the interaction of SM fields with the KK modes of the gravity. To lowest order in $\kappa$, the KK modes couple to SM fields through energy momentum tensor of SM. Here, we restrict ourselves to QCD part of the energy momentum tensor:

$$
\mathcal{S}=\mathcal{S}_{S M}-\frac{\kappa}{2} \int d^{4} x T_{\mu \nu}^{Q C D}(x) h^{\mu \nu}(x),
$$

where $T_{\mu \nu}^{Q C D}$ is the energy momentum tensor of QCD [10-12]:

$$
\begin{aligned}
T_{\mu \nu}^{Q C D}= & -g_{\mu \nu} \mathcal{L}_{Q C D}-F_{\mu \rho}^{a} F_{\nu}^{a \rho}-\frac{1}{\xi} g_{\mu \nu} \partial^{\rho}\left(A_{\rho}^{a} \partial^{\sigma} A_{\sigma}^{a}\right) \\
& +\frac{1}{\xi}\left(A_{\nu}^{a} \partial_{\mu}\left(\partial^{\sigma} A_{\sigma}^{a}\right)+A_{\mu}^{a} \partial_{\nu}\left(\partial^{\sigma} A_{\sigma}^{a}\right)\right)+\frac{i}{4}\left[\bar{\psi} \gamma_{\mu}\left(\vec{\partial}_{\nu}-i g_{s} T^{a} A_{\nu}^{a}\right) \psi\right. \\
& -\bar{\psi}\left(\overleftarrow{\partial}_{\nu}+i g_{s} T^{a} A_{\nu}^{a}\right) \gamma_{\mu} \psi+\bar{\psi} \gamma_{\nu}\left(\vec{\partial}_{\mu}-i g_{s} T^{a} A_{\mu}^{a}\right) \psi \\
& \left.-\bar{\psi}\left(\overleftarrow{\partial}_{\mu}+i g_{s} T^{a} A_{\mu}^{a}\right) \gamma_{\nu} \psi\right]+\partial_{\mu} \bar{\omega}^{a}\left(\partial_{\nu} \omega^{a}-g_{s} f^{a b c} A_{\nu}^{c} \omega^{b}\right) \\
& +\partial_{\nu} \bar{\omega}^{a}\left(\partial_{\mu} \omega^{a}-g_{s} f^{a b c} A_{\mu}^{c} \omega^{b}\right) .
\end{aligned}
$$

In the above equation, $g_{s}$ is the strong coupling constant and $\xi$ is gauge parameter in Lorenz gauge fixing condition. The peculiar feature in the above action is the appearance of the direct coupling of ghost fields $(\omega, \bar{\omega})$ with KK modes [10-12]. We have kept track of these unphysical contributions along with those coming from gauge fixing term in order to establish the cancellation of their contributions among themselves. We have retained only light flavours in the quark sector.

We compute the relevant form factors by evaluating the truncated matrix elements $\hat{\mathcal{M}}_{I}$ of $T_{\mu \nu}^{Q C D}$ between on-shell gluon $(I=g)$ and quark/anti-quark $(I=q, \bar{q})$ states. The symbol ^ here and in the following denotes that the quantities are unrenormalised/bare. The $\hat{\mathcal{M}}_{I}$ s in the color space can be expanded as

$$
\hat{\mathcal{M}}_{I}=\hat{\mathcal{M}}_{I}^{(0)}+\hat{a}_{s}\left(\frac{Q^{2}}{\mu^{2}}\right)^{\frac{\varepsilon}{2}} S_{\varepsilon} \hat{\mathcal{M}}_{I}^{(1)}+\hat{a}_{s}^{2}\left(\frac{Q^{2}}{\mu^{2}}\right)^{\varepsilon} S_{\varepsilon}^{2} \hat{\mathcal{M}}_{I}^{(2)}+\mathcal{O}\left(\hat{a}_{s}^{3}\right), I=g, q, \bar{q},
$$


where the unrenormalised coupling constant $\hat{a}_{s}=\hat{g}_{s}^{2} / 16 \pi^{2}$ and the scale $\mu$ is introduced to keep $g_{s}$ dimensionless in dimensional regularisation and the space-time dimension is taken to be $d=4+\varepsilon$. The scale $Q^{2}=-q^{2}-i \epsilon$, where $q$ is the momentum transfer. The unrenormalised coupling constant $\hat{a}_{s}$ is related to the renormalised one $a_{s}\left(\mu_{R}^{2}\right)$ by

$$
S_{\varepsilon} \hat{a}_{s}=Z\left(\mu_{R}^{2}\right) a_{s}\left(\mu_{R}^{2}\right)\left(\frac{\mu^{2}}{\mu_{R}^{2}}\right)^{\frac{\varepsilon}{2}}, \quad S_{\varepsilon}=\exp \left\{\frac{\varepsilon}{2}\left[\gamma_{E}-\ln 4 \pi\right]\right\}
$$

where the renormalisation constant $Z\left(\mu_{R}^{2}\right)$ is given by

$$
Z\left(\mu_{R}^{2}\right)=1+a_{s}\left(\mu_{R}^{2}\right) \frac{2 \beta_{0}}{\varepsilon}+\mathcal{O}\left(a_{s}^{2}\left(\mu_{R}^{2}\right)\right), \quad \beta_{0}=\frac{11}{3} C_{A}-\frac{4}{3} T_{F} n_{f}
$$

with $\mu_{R}$-renormalisation scale, $C_{A}=N, T_{F}=1 / 2$ and $n_{f}$ the number of active flavours.

Using the $\hat{\mathcal{M}}_{I}$ s, the form factors are defined as

$$
\hat{F}_{I}^{T,(n)}=\frac{\hat{\mathcal{M}}_{I}^{(0) *} \cdot \hat{\mathcal{M}}_{I}^{(n)}}{\hat{\mathcal{M}}_{I}^{(0) *} \cdot \hat{\mathcal{M}}_{I}^{(0)}},
$$

and the symbol · takes care of the color and spin/polarisation sums.

The Feynman amplitudes that contribute to gluon and quark matrix elements of the energy momentum tensor $T_{\mu \nu}^{Q C D}$ at born, one-loop and two-loop levels in QCD are obtained using a computer program QGRAF [20]. We find 12 one-loop and 153 two-loop diagrams that contribute to the matrix element of energy momentum tensor if it is computed between gluon states while 4 one-loop and 54 two-loop diagrams that contribute for quark-antiquark states. We have used a set of in-house FORM [21] routines to convert the QGRAF outputs into a suitable form for further symbolic manipulations. These FORM routines not only replace the symbolic Feynman vertices, propagators by the corresponding Feynman rules but also perform Lorentz contractions, Dirac gamma matrix algebra etc. We have done all our computations in $d=4+\varepsilon$ dimensions in order to regulate both ultraviolet (UV) and infrared (IR) divergences. The resulting expressions at this stage contain one and two-loop tensor and scalar integrals. Since the coupling of KK modes with the energy momentum tensor involves quadratic derivatives, we find that the rank of the tensor integrals present in our computation is larger than the rank of integrals appearing in quark and gluon form factors contributing to electroweak vector boson [22-25] and Higgs production cross sections (in the infinite top quark mass limit) [26-29] respectively.

In the past, they were computed using very different methods, that is, different techniques were employed to perform loop integrals. In [22], the method of Feynman parameterisation was used in a judicious way so that after each parametric integration one is left with an integral over the next parameter. In [23], an elegant method, advocated in [30, 31], namely "integration by parts" (IBP) was used. In [24, 25] the integrals were computed using dispersion techniques developed in [32] which uses the Cutkosky rules [33]. In this method, one cuts the Feynman amplitude in all possible ways to obtain the imaginary part and the real part was obtained from the imaginary part via a dispersion relation. In [26], an algorithm [34] which relates $l$-loop integrals with $n+1$ external legs to $l+1$-loop integrals 
with $n$ external legs was used to compute two-loop QCD corrections to gluon form factor relevant for Higgs production cross section. It maps the massless two-loop vertex functions onto massless three-loop two-point functions which are relatively easy to compute. In [27], IBP identities were used extensively to compute the gluon form factor. The gluon form factor at two-loop level in $\mathrm{SU}(N)$ gauge theory with $n_{f}$ light flavours was computed in [29] following [32]. All these results were known only to a desired accuracy in $\varepsilon$, say $\mathcal{O}(\varepsilon)$. In [35], using IBP [30, 31] and Lorentz invariance (LI) [36] identities, the authors have shown that the two-loop corrections to electroweak quark and gluon form factors can be expressed in terms of only few master integrals and have also obtained for the first time the closed form solution to one of the master integrals whose result was known only up to few orders in $\varepsilon$. We will closely follow this approach by [35] to achieve our task. Reduction of a large number of one and two-loop tensor integrals that appear in our computation to a few master integrals was achieved by FIRE [37], a Mathematica package, which extensively uses the IBP [30, 31] and LI [36] identities implemented using Laporta algorithm [38]. Note that there are also similar packages namely AIR [39], Reduce [40, 41] and most recently LiteRed [42] that can do this reduction. We used LiteRed to cross-check our results obtained using FIRE. At one-loop level, we find that the form factors depend only on one master integral and at two-loop level, there are one one-loop and three two-loop master integrals. These one-loop and two-loop master integrals are now known to all orders in $\varepsilon$ and are given in [35]. Below we present our final results for $\hat{F}_{I}^{T,(n)}$ for $I=g, q ; n=1,2$ in terms of these master integrals.

For the gluon form factor, we obtain $\hat{F}_{g}^{T,(0)}=1$ and

$$
\begin{aligned}
\hat{F}_{g}^{T,(1)}= & 2\left[-i A_{2, L O}\left(4 C_{A}\left(-68+20 d+16 d^{2}-8 d^{3}+d^{4}\right)\right.\right. \\
& \left.\left.+n_{f}\left(-16+32 d-15 d^{2}+2 d^{3}\right)\right)\right] /\left[(-4+d)(-2+d)\left(2 d^{2}-3 d-8\right)\right], \\
\hat{F}_{g}^{T,(2)}= & -\left[16 A_{2, L O}^{2}\left(-3360+5524 d-3607 d^{2}+1169 d^{3}-188 d^{4}+12 d^{5}\right)\right. \\
& \times\left\{4 C _ { A } ^ { 2 } \left(384+3584 d-7712 d^{2}+4260 d^{3}+128 d^{4}-939 d^{5}+371 d^{6}-62 d^{7}\right.\right. \\
& \left.+4 d^{8}\right)+C_{F} d\left(1024-3616 d+4720 d^{2}-2926 d^{3}+941 d^{4}-153 d^{5}+10 d^{6}\right) n_{f} \\
& \left.+8 C_{A}\left(192-288 d-28 d^{2}+322 d^{3}-255 d^{4}+89 d^{5}-15 d^{6}+d^{7}\right) n_{f}\right\} \\
& +A_{3}(-8+3 d)\left\{2 C _ { A } ^ { 2 } \left(1720320+60414976 d-195105152 d^{2}+236351744 d^{3}\right.\right. \\
& -120445352 d^{4}-11375804 d^{5}+54553314 d^{6}-36985777 d^{7}+13961672 d^{8} \\
& \left.-3324848 d^{9}+499154 d^{10}-43447 d^{11}+1680 d^{12}\right)+2 C_{F} d(10379264 \\
& -36831232 d+50367872 d^{2}-28580992 d^{3}-3473320 d^{4}+16083820 d^{5}
\end{aligned}
$$




$$
\begin{aligned}
& \left.-11518542 d^{6}+4520247 d^{7}-1098971 d^{8}+165551 d^{9}-14233 d^{10}+536 d^{11}\right) n_{f} \\
& +C_{A}\left(3440640+10901504 d-45510400 d^{2}+62792448 d^{3}-46643440 d^{4}\right. \\
& +20064592 d^{5}-4109776 d^{6}-494472 d^{7}+619031 d^{8}-203281 d^{9}+36557 d^{10} \\
& \left.\left.-3641 d^{11}+158 d^{12}\right) n_{f}\right\}+2\left(12-7 d+d^{2}\right)\left(2 A_{6}(-4+d)^{2} d(-16+30 d\right. \\
& \left.-17 d^{2}+3 d^{3}\right)\left\{C_{A}^{2}\left(3392-3664 d+284 d^{2}+794 d^{3}-298 d^{4}+32 d^{5}\right)\right. \\
& +2 C_{F}(-4+d)^{2}\left(176-26 d-35 d^{2}+8 d^{3}\right) n_{f}+C_{A}\left(-2880+1888 d+172 d^{2}\right. \\
& \left.\left.-368 d^{3}+87 d^{4}-6 d^{5}\right) n_{f}\right\}+A_{4}\left\{2 C_{A}^{2}(-1720320+25437184 d\right. \\
& -55822976 d^{2}+41289728 d^{3}+1696440 d^{4}-22168812 d^{5}+16330266 d^{6} \\
& \left.-6288301 d^{7}+1498316 d^{8}-234230 d^{9}+24945 d^{10}-1812 d^{11}+72 d^{12}\right) \\
& +2 C_{F}(-4+d)^{2} d\left(-479744+1418752 d-1664968 d^{2}+989740 d^{3}\right. \\
& \left.-297578 d^{4}+28179 d^{5}+7786 d^{6}-2351 d^{7}+184 d^{8}\right) n_{f}+C_{A}(-3440640 \\
& +18589696 d-42184960 d^{2}+55760640 d^{3}-47369168 d^{4}+26855488 d^{5} \\
& -10323440 d^{6}+2698144 d^{7}-474715 d^{8}+54662 d^{9}-3837 d^{10} \\
& \left.\left.\left.\left.+130 d^{11}\right) n_{f}\right\}\right)\right] /\left[8(-4+d)^{3}(-3+d)(-2+d)^{2}(-1+d) d(-7+2 d)\right. \\
& \left.\times(-5+2 d)(-8+3 d)\left(2 d^{2}-3 d-8\right)\right] \text {, }
\end{aligned}
$$

where the color factor $C_{F}=\left(N^{2}-1\right) / 2 N$. For the quark form factor, we obtain $\hat{F}_{q}^{T,(0)}=1$ and

$$
\begin{aligned}
\hat{F}_{q}^{T,(1)}= & 2\left[-\frac{i}{2} A_{2, L O} C_{F}\left(64-34 d+5 d^{2}\right)\right] /[(d-4)(d-2)] \\
\hat{F}_{q}^{T,(2)}= & -\left[C _ { F } \left(16 A_{2, L O}^{2}\left(-3360+5524 d-3607 d^{2}+1169 d^{3}-188 d^{4}+12 d^{5}\right)\right.\right. \\
& \times\left\{C_{F} d\left(2048-5312 d+5156 d^{2}-2432 d^{3}+619 d^{4}-84 d^{5}+5 d^{6}\right)\right. \\
& +16 C_{A}\left(192-288 d-28 d^{2}+322 d^{3}-255 d^{4}+89 d^{5}-15 d^{6}+d^{7}\right) \\
& \left.+4(-4+d)^{2}\left(48-56 d+35 d^{2}-13 d^{3}+2 d^{4}\right) n_{f}\right\}+A_{3}(-8+3 d) \\
& \times\left\{C _ { A } \left(6881280-11370496 d-5231104 d^{2}+24600896 d^{3}-18172384 d^{4}\right.\right. \\
& -2105928 d^{5}+11581460 d^{6}-8688682 d^{7}+3558513 d^{8}-910210 d^{9}
\end{aligned}
$$




$$
\begin{aligned}
& \left.+146166 d^{10}-13592 d^{11}+561 d^{12}\right)-2\left(C_{F} d(-13110272+59524736 d\right. \\
& -119256896 d^{2}+139910176 d^{3}-107260248 d^{4}+56580636 d^{5}-20992430 d^{6} \\
& \left.+5484477 d^{7}-989746 d^{8}+117582 d^{9}-8276 d^{10}+261 d^{11}\right)-16(-4+d)^{2} \\
& \times\left(13440-1936 d-63236 d^{2}+124494 d^{3}-119835 d^{4}+68959 d^{5}\right. \\
& \left.\left.\left.-24789 d^{6}+5463 d^{7}-676 d^{8}+36 d^{9}\right) n_{f}\right)\right\}+2\left(-12+19 d-8 d^{2}+d^{3}\right) \\
& \times\left(2 A _ { 6 } ( - 4 + d ) ^ { 2 } d ( - 1 6 + 3 0 d - 1 7 d ^ { 2 } + 3 d ^ { 3 } ) \left\{2 C_{F}(-288+192 d\right.\right. \\
& \left.\left.-16 d^{2}-6 d^{3}+d^{4}\right)+C_{A}\left(416-192 d+24 d^{2}-14 d^{3}+3 d^{4}\right)\right\} \\
& +A_{4}\left\{C _ { A } \left(6881280-23730176 d+37309440 d^{2}-32039680 d^{3}\right.\right. \\
& +15239072 d^{4}-3272584 d^{5}-345324 d^{6}+415114 d^{7}-116931 d^{8}+17142 d^{9} \\
& \left.-1361 d^{10}+48 d^{11}\right)-2\left(C _ { F } d \left(677888-2026112 d+2909696 d^{2}\right.\right. \\
& -2895040 d^{3}+2126552 d^{4}-1101532 d^{5}+384546 d^{6}-87351 d^{7}+12286 d^{8} \\
& \left.-965 d^{9}+32 d^{10}\right)-4\left(860160-1270784 d+218048 d^{2}+766736 d^{3}\right. \\
& -743952 d^{4}+330352 d^{5}-81952 d^{6}+10967 d^{7}-533 d^{8}-36 d^{9} \\
& \left.\left.\left.\left.\left.+4 d^{10}\right) n_{f}\right)\right\}\right)\right) /\left[16(-4+d)^{3}(-3+d)(-2+d)^{2}(-1+d)^{2} d\right. \\
& \times(-7+2 d)(-5+2 d)(-8+3 d)]
\end{aligned}
$$

The exact results for the master integrals $A_{i}(i=\{2, L O\}, 3,4,6)$ can be expressed in terms of Euler Gamma functions and are available in the works on two-loop electroweak form factors [35]. The most difficult crossed two-loop master integral $A_{6}$ was solved exactly in [35]. These results are used to present the form factors to order $\mathcal{O}\left(\varepsilon^{4}\right)$ and are given in the appendix. We use them to study the infrared pole structure of these farm factors in the next section. The higher order terms $\mathcal{O}\left(\varepsilon^{i}\right), i>0$ are also useful to perform ultraviolet renormalisation of the form factors beyond two-loop level.

\section{Infrared divergence structure}

Having obtained these form factors at two-loop level, the next step is to study the infrared pole structure of these factors in order to establish the universal behaviour of these QCD amplitudes. In the past, there have been detailed studies of quark and gluon form factors through Sudakov integro-differential equation [43-47], see also [29, 48-51]. Since the KK modes are colour singlet fields, the unrenormalised form factors $\hat{F}_{I}^{T}\left(\hat{a}_{s}, Q^{2}, \mu^{2}, \varepsilon\right)$ are expected to satisfy similar integro-differential equation that follows from the gauge as well as 
renormalisation group $(\mathrm{RG})$ invariances. In dimensional regularisation,

$$
Q^{2} \frac{d}{d Q^{2}} \ln \hat{F}_{I}^{T}\left(\hat{a}_{s}, Q^{2}, \mu^{2}, \varepsilon\right)=\frac{1}{2}\left[K^{T, I}\left(\hat{a}_{s}, \frac{\mu_{R}^{2}}{\mu^{2}}, \varepsilon\right)+G^{T, I}\left(\hat{a}_{s}, \frac{Q^{2}}{\mu_{R}^{2}}, \frac{\mu_{R}^{2}}{\mu^{2}}, \varepsilon\right)\right]
$$

where the constants $K^{T, I}$ contain all the poles in $\varepsilon$, and $G^{T, I}$ are finite as $\varepsilon$ becomes zero. The RG invariance of $\hat{F}_{I}^{T}$ gives

$$
\begin{aligned}
\mu_{R}^{2} \frac{d}{d \mu_{R}^{2}} K^{T, I}\left(\hat{a}_{s}, \frac{\mu_{R}^{2}}{\mu^{2}}, \varepsilon\right) & =-A^{T, I}\left(a_{s}\left(\mu_{R}^{2}\right)\right), \\
\mu_{R}^{2} \frac{d}{d \mu_{R}^{2}} G^{T, I}\left(\hat{a}_{s}, \frac{Q^{2}}{\mu_{R}^{2}}, \frac{\mu_{R}^{2}}{\mu^{2}}, \varepsilon\right) & =A^{T, I}\left(a_{s}\left(\mu_{R}^{2}\right)\right) .
\end{aligned}
$$

The quantities $A^{T, I}$ are the cusp anomalous dimensions which are expanded as

$$
A^{T, I}\left(a_{s}\left(\mu_{R}^{2}\right)\right)=\sum_{i=1}^{\infty} a_{s}^{i}\left(\mu_{R}^{2}\right) A_{i}^{T, I} .
$$

Solving these RG equations, the constants $K^{T, I}$ and $G^{T, I}$ can be obtained in powers of bare coupling constant $\hat{a}_{s}$. Using these solutions, we obtain,

$$
\ln \hat{F}_{I}^{T}\left(\hat{a}_{s}, Q^{2}, \mu^{2}, \varepsilon\right)=\sum_{i=1}^{\infty} \hat{a}_{s}^{i}\left(\frac{Q^{2}}{\mu^{2}}\right)^{i \frac{\varepsilon}{2}} S_{\varepsilon}^{i} \hat{\mathcal{L}}_{F^{T}}^{I(i)}(\varepsilon),
$$

where

$$
\begin{aligned}
& \hat{\mathcal{L}}_{F^{T}}^{I(1)}=\frac{1}{\varepsilon^{2}}\left(-2 A_{1}^{T, I}\right)+\frac{1}{\varepsilon}\left(G_{1}^{T, I}(\varepsilon)\right) \\
& \hat{\mathcal{L}}_{F^{T}}^{I(2)}=\frac{1}{\varepsilon^{3}}\left(\beta_{0} A_{1}^{T, I}\right)+\frac{1}{\varepsilon^{2}}\left(-\frac{1}{2} A_{2}^{T, I}-\beta_{0} G_{1}^{T, I}(\varepsilon)\right)+\frac{1}{2 \varepsilon} G_{2}^{T, I}(\varepsilon) .
\end{aligned}
$$

The cusp anomalous dimensions $A_{i}^{T, I}$ can be obtained by comparing eqs. (3.4), (3.5) and the results of the form factors, eqns (A.1), (A.2), (A.3), (A.4). We find that they are identical to those obtained in [52], that is, those appearing in gluon and quark form factors, confirming the universality of IR structure of these form factors. The coefficients $G_{i}^{T, I}(\varepsilon)$ take the following form

$$
\begin{aligned}
& G_{1}^{T, I}(\varepsilon)=2 B_{1}^{T, I}+f_{1}^{T, I}+\sum_{k=1}^{\infty} \varepsilon^{k} g_{1}^{T, I, k} \\
& G_{2}^{T, I}(\varepsilon)=2 B_{2}^{T, I}+f_{2}^{T, I}-2 \beta_{0} g_{1}^{T, I, 1}+\sum_{k=1}^{\infty} \varepsilon^{k} g_{2}^{T, I, k},
\end{aligned}
$$

where again the collinear anomalous dimension $B_{i}^{T, I}$ and soft anomalous dimension $f_{i}^{T, I}$ are found to be identical to $B_{i}^{I}$ and $f_{i}^{I}$ obtained in $[29,53]$ for quark and gluon form factors. We find that only $g_{i}^{T, I, k}$ are operator dependent. 
Another independent check on our computation is done by establishing the connection between these form factors and the very successful proposal by Catani [54] (also see [55]) on one and two-loop QCD amplitudes using the universal factors $\mathbf{I}_{I}^{(i)}(\varepsilon)$ and $\mathbf{H}_{I}^{(i)}, i=1,2$. The all order generalisation of Catani's proposal was obtained by Becher and Neubert [56] and also by Gardi and Magnea [57]. These universal factors capture all the IR poles of n-parton QCD amplitudes up to two-loop level in QCD. Following [54], we proceed by expressing the matrix elements in terms of UV renormalised ones as

$$
\hat{\mathcal{M}}_{I}=\mathbf{M}_{I}^{(0)}+a_{s}\left(\mu_{R}^{2}\right) \mathbf{M}_{I}^{(1)}+a_{s}^{2}\left(\mu_{R}^{2}\right) \mathbf{M}_{I}^{(2)}+\mathcal{O}\left(a_{s}^{3}\left(\mu_{R}^{2}\right)\right), \quad I=g, q, \bar{q} .
$$

Using the universal $\mathbf{I}_{I}(\varepsilon)$ obtained by Catani, we can write down

$$
\begin{aligned}
& \mathbf{M}_{I}^{(1)}=2 \mathbf{I}_{I}^{(1)}(\varepsilon) \mathbf{M}_{I}^{(0)}(\varepsilon)+\mathbf{M}_{I, \text { fin }}^{(1)}(\varepsilon) \\
& \mathbf{M}_{I}^{(2)}=2 \mathbf{I}_{I}^{(1)}(\varepsilon) \mathbf{M}_{I}^{(1)}(\varepsilon)+4 \mathbf{I}_{I}^{(2)}(\varepsilon) \mathbf{M}_{I}^{(0)}(\varepsilon)+\mathbf{M}_{I, \text { fin }}^{(2)}(\varepsilon)
\end{aligned}
$$

In terms of these $\mathbf{M}_{I}^{(i)}$, we find

$$
\begin{aligned}
\hat{F}_{I}^{T,(1)}=2 \mu_{R}^{\varepsilon} \mathbf{I}_{I}^{(1)}(\varepsilon)+\hat{F}_{I, \text { fin }}^{T,(1)}(\varepsilon), & \\
\hat{F}_{I}^{T,(2)}=4 \mu_{R}^{2 \varepsilon}[ & \left(\mathbf{I}_{I}^{(1)}(\varepsilon)\right)^{2}+\mathbf{I}_{I}^{(2)}(\varepsilon)-\frac{\beta_{0}}{\varepsilon}\left(\mathbf{I}_{I}^{(1)}(\varepsilon)+\frac{\mu_{R}^{-\varepsilon}}{2} \hat{F}_{I, \text { fin }}^{T,(1)}(\varepsilon)\right) \\
& \left.+\frac{1}{2} \mu_{R}^{-\varepsilon} \mathbf{I}_{I}^{(1)}(\varepsilon) \hat{F}_{I, \text { fin }}^{T,(1)}(\varepsilon)\right]+\hat{F}_{I, \text { fin }}^{T,(2)}(\varepsilon),
\end{aligned}
$$

where

$$
\hat{F}_{I, \text { fin }}^{T,(i)}(\varepsilon)=\mu_{R}^{i \varepsilon} \frac{\mathbf{M}_{I}^{(0) *} \cdot \mathbf{M}_{I, \text { fin }}^{(i)}}{\mathbf{M}_{I}^{(0) *} \cdot \mathbf{M}_{I}^{(0)}}, \quad i=1,2
$$

The singular universal functions $\mathbf{I}_{I}^{(i)}$ are given by

$$
\begin{aligned}
\mathbf{I}_{q}^{(1)}(\varepsilon)= & -\frac{e^{-\varepsilon \gamma_{E} / 2}}{\Gamma\left(1+\frac{\varepsilon}{2}\right)}\left(\frac{Q^{2}}{\mu_{R}^{2}}\right)^{\frac{\varepsilon}{2}}\left(4 \frac{C_{F}}{\varepsilon^{2}}-3 \frac{C_{F}}{\varepsilon}\right), \\
\mathbf{I}_{g}^{(1)}(\varepsilon)= & -\frac{e^{-\varepsilon \gamma_{E} / 2}}{\Gamma\left(1+\frac{\varepsilon}{2}\right)}\left(\frac{Q^{2}}{\mu_{R}^{2}}\right)^{\frac{\varepsilon}{2}}\left(4 \frac{C_{A}}{\varepsilon^{2}}-\frac{\beta_{0}}{\varepsilon}\right), \\
\mathbf{I}_{I}^{(2)}(\varepsilon)= & -\frac{1}{2}\left(\mathbf{I}_{I}^{(1)}(\varepsilon)\right)^{2}+\frac{\beta_{0}}{\varepsilon} \mathbf{I}_{I}^{(1)}(\varepsilon) \\
& +\frac{e^{\frac{\varepsilon \gamma_{E}}{2}} \Gamma(1+\varepsilon)}{\Gamma\left(1+\frac{\varepsilon}{2}\right)}\left(-\frac{\beta_{0}}{\varepsilon}+K\right) \mathbf{I}_{I}^{(1)}(2 \varepsilon)+\mathbf{H}_{I}^{(2)} \frac{1}{\varepsilon},
\end{aligned}
$$

and

$$
K=\left(\frac{67}{18}-\zeta_{2}\right) C_{A}-\frac{10}{9} T_{F} n_{f}
$$


Using our results for $\hat{F}^{T,(i)}$ given in eqs. (A.1), (A.2), (A.3), (A.4) and the results for $\mathbf{I}_{I}^{(i)}$ given in [54], we obtain $\mathbf{H}_{I}^{(2)}$ :

$$
\begin{aligned}
& \mathbf{H}_{g}^{(2)}=C_{A}^{2}\left(-\frac{5}{12}-\frac{11}{24} \zeta_{2}-\frac{1}{2} \zeta_{3}\right)+C_{A} n_{f}\left(\frac{29}{27}+\frac{1}{12} \zeta_{2}\right)+C_{F} n_{f}\left(-\frac{1}{2}\right)+n_{f}^{2}\left(-\frac{5}{27}\right), \\
& \mathbf{H}_{q}^{(2)}=C_{F}^{2}\left(\frac{3}{8}-3 \zeta_{2}+6 \zeta_{3}\right)+C_{A} C_{F}\left(-\frac{245}{216}+\frac{23}{8} \zeta_{2}-\frac{13}{2} \zeta_{3}\right)+C_{F} n_{f}\left(\frac{25}{108}-\frac{1}{4} \zeta_{2}\right) \cdot(3.14)
\end{aligned}
$$

The single pole coefficients thus obtained agree with the color diagonal part of eq. (12) of [56] (see also eq. (4.21) of [29] for quark and gluon form factors and [58-61] for four parton amplitudes). This serves as a check on our computation and also establishes the proposal by Catani on IR universality of QCD amplitudes with $T_{\mu \nu}$ insertion.

\section{Conclusions}

We present an important ingredient to the full NNLO QCD correction to graviton mediated hadronic scattering processes namely the gluon and quark form factors of energy momentum tensor of the QCD part of the SM up to two-loop level in QCD. We have used dimensional regularisation to obtain these form factors in $\mathrm{SU}(N)$ gauge theory with $n_{f}$ light flavours. Both exact as well as expanded results in $\varepsilon$ are presented. The higher order terms in $\varepsilon$ of these form factors are important for the ultraviolet renormalisation of these amplitudes at three-loop level. We have shown that these form factors satisfy Sudakov integro-differential equation with same cusp $A_{I}$, collinear $B^{I}$ and soft $f^{I}$ anomalous dimensions that contribute to electroweak vector boson and gluon form factors. In addition, they also show the universal behaviour of the infrared poles in $\varepsilon$ in accordance with the proposal by Catani.

Spin-2 resonance production has been widely studied in the context of the Higgs [62] and BSM models [63]. The two-loop results presented in this paper would further reduce the theoretical uncertainties and hence improve the predictions in disentangling the various postulates. We further plan to apply these two-loop results to the $\mathrm{TeV}$ scale gravity models [64].

\section{Acknowledgments}

The work of DdeF and JM was supported in part by UBACYT, CONICET, ANPCyT and the Research Executive Agency (REA) of the European Union under the Grant Agreement number PITN-GA-2010-264564 (LHCPhenoNet). MM would like to thank IMSc for hospitality. 


\section{A $\varepsilon$ expansion of the two-loop form factors}

We present here the form factors as a series expansion in $\varepsilon$ up to $\mathcal{O}\left(\varepsilon^{4}\right)$ for $F_{I}^{(1)}$ and up to $\mathcal{O}\left(\varepsilon^{2}\right)$ for $F_{I}^{(2)}$ :

$$
\begin{aligned}
& \hat{F}_{g}^{T,(1)}=n_{f}\left[\frac{1}{\varepsilon}\left(-\frac{4}{3}\right)+\left(\frac{35}{18}\right)+\varepsilon\left(-\frac{497}{216}+\frac{1}{6} \zeta_{2}\right)+\varepsilon^{2}\left(\frac{6593}{2592}-\frac{7}{18} \zeta_{3}-\frac{35}{144} \zeta_{2}\right)\right. \\
& +\varepsilon^{3}\left(-\frac{84797}{31104}+\frac{245}{432} \zeta_{3}+\frac{497}{1728} \zeta_{2}+\frac{47}{480} \zeta_{2}^{2}\right)+\varepsilon^{4}\left(\frac{1072433}{373248}-\frac{31}{120} \zeta_{5}\right. \\
& \left.\left.-\frac{3479}{5184} \zeta_{3}-\frac{6593}{20736} \zeta_{2}+\frac{7}{144} \zeta_{2} \zeta_{3}-\frac{329}{2304} \zeta_{2}^{2}\right)\right]+C_{A}\left[\frac{1}{\varepsilon^{2}}(-8)+\frac{1}{\varepsilon}\left(\frac{22}{3}\right)\right. \\
& +\left(-\frac{203}{18}+\zeta_{2}\right)+\varepsilon\left(\frac{2879}{216}-\frac{7}{3} \zeta_{3}-\frac{11}{12} \zeta_{2}\right)+\varepsilon^{2}\left(-\frac{37307}{2592}+\frac{77}{36} \zeta_{3}+\frac{203}{144} \zeta_{2}\right. \\
& \left.+\frac{47}{80} \zeta_{2}^{2}\right)+\varepsilon^{3}\left(\frac{465143}{31104}-\frac{31}{20} \zeta_{5}-\frac{1421}{432} \zeta_{3}-\frac{2879}{1728} \zeta_{2}+\frac{7}{24} \zeta_{2} \zeta_{3}-\frac{517}{960} \zeta_{2}^{2}\right) \\
& +\varepsilon^{4}\left(-\frac{5695811}{373248}+\frac{341}{240} \zeta_{5}+\frac{20153}{5184} \zeta_{3}-\frac{49}{144} \zeta_{3}^{2}+\frac{37307}{20736} \zeta_{2}-\frac{77}{288} \zeta_{2} \zeta_{3}\right. \\
& \left.\left.+\frac{9541}{11520} \zeta_{2}^{2}+\frac{949}{4480} \zeta_{2}^{3}\right)\right] \\
& \hat{F}_{g}^{T,(2)}=C_{F} n_{f}\left[\frac{1}{\varepsilon}(-2)+\left(\frac{61}{6}-8 \zeta_{3}\right)+\varepsilon\left(-\frac{2245}{72}+\frac{59}{3} \zeta_{3}+\frac{1}{2} \zeta_{2}+\frac{12}{5} \zeta_{2}^{2}\right)\right. \\
& +\varepsilon^{2}\left(\frac{64177}{864}-14 \zeta_{5}-\frac{335}{9} \zeta_{3}-\frac{83}{24} \zeta_{2}+2 \zeta_{2} \zeta_{3}-\frac{179}{30} \zeta_{2}^{2}\right)+C_{A} n_{f}\left[\frac{1}{\varepsilon^{3}}(8)\right. \\
& +\frac{1}{\varepsilon^{2}}\left(-\frac{40}{3}\right)+\frac{1}{\varepsilon}\left(\frac{41}{3}-\frac{2}{3} \zeta_{2}\right)+\left(-\frac{605}{108}+10 \zeta_{3}+\frac{5}{9} \zeta_{2}\right)+\varepsilon\left(-\frac{21557}{1296}\right. \\
& \left.-\frac{182}{9} \zeta_{3}+\frac{145}{108} \zeta_{2}-\frac{57}{20} \zeta_{2}^{2}\right)+\varepsilon^{2}\left(\frac{320813}{5184}+\frac{71}{10} \zeta_{5}+\frac{6407}{216} \zeta_{3}-\frac{3617}{648} \zeta_{2}\right. \\
& \left.-\frac{43}{18} \zeta_{2} \zeta_{3}+\frac{1099}{180} \zeta_{2}^{2}\right)+C_{A}^{2}\left[\frac{1}{\varepsilon^{4}}(32)+\frac{1}{\varepsilon^{3}}(-44)+\frac{1}{\varepsilon^{2}}\left(\frac{226}{3}-4 \zeta_{2}\right)\right. \\
& +\frac{1}{\varepsilon}\left(-81+\frac{50}{3} \zeta_{3}+\frac{11}{3} \zeta_{2}\right)+\left(\frac{5249}{108}-11 \zeta_{3}-\frac{67}{18} \zeta_{2}-\frac{21}{5} \zeta_{2}^{2}\right)+\varepsilon\left(\frac{59009}{1296}\right. \\
& \left.-\frac{71}{10} \zeta_{5}+\frac{433}{18} \zeta_{3}-\frac{337}{108} \zeta_{2}-\frac{23}{6} \zeta_{2} \zeta_{3}+\frac{99}{40} \zeta_{2}^{2}\right)+\varepsilon^{2}\left(-\frac{1233397}{5184}+\frac{759}{20} \zeta_{5}\right. \\
& \left.\left.-\frac{8855}{216} \zeta_{3}+\frac{901}{36} \zeta_{3}^{2}+\frac{12551}{648} \zeta_{2}+\frac{77}{36} \zeta_{2} \zeta_{3}-\frac{4843}{720} \zeta_{2}^{2}+\frac{2313}{280} \zeta_{2}^{3}\right)\right]
\end{aligned}
$$




$$
\begin{aligned}
& \hat{F}_{q}^{T,(1)}=C_{F}\left[\frac{1}{\varepsilon^{2}}(-8)+\frac{1}{\varepsilon}(6)+\left(-10+\zeta_{2}\right)+\varepsilon\left(12-\frac{7}{3} \zeta_{3}-\frac{3}{4} \zeta_{2}\right)\right. \\
& +\varepsilon^{2}\left(-13+\frac{7}{4} \zeta_{3}+\frac{5}{4} \zeta_{2}+\frac{47}{80} \zeta_{2}^{2}\right)+\varepsilon^{3}\left(\frac{27}{2}-\frac{31}{20} \zeta_{5}-\frac{35}{12} \zeta_{3}-\frac{3}{2} \zeta_{2}\right. \\
& \left.+\frac{7}{24} \zeta_{2} \zeta_{3}-\frac{141}{320} \zeta_{2}^{2}\right)+\varepsilon^{4}\left(-\frac{55}{4}+\frac{93}{80} \zeta_{5}+\frac{7}{2} \zeta_{3}-\frac{49}{144} \zeta_{3}^{2}+\frac{13}{8} \zeta_{2}\right. \\
& \left.\left.-\frac{7}{32} \zeta_{2} \zeta_{3}+\frac{47}{64} \zeta_{2}^{2}+\frac{949}{4480} \zeta_{2}^{3}\right)\right] \\
& \hat{F}_{q}^{T,(2)}=C_{F} n_{f}\left[\frac{1}{\varepsilon^{3}}\left(-\frac{8}{3}\right)+\frac{1}{\varepsilon^{2}}\left(\frac{56}{9}\right)+\frac{1}{\varepsilon}\left(-\frac{425}{27}-\frac{2}{3} \zeta_{2}\right)+\left(\frac{9989}{324}-\frac{26}{9} \zeta_{3}+\frac{38}{9} \zeta_{2}\right)\right. \\
& +\varepsilon\left(-\frac{202253}{3888}+\frac{2}{27} \zeta_{3}-\frac{989}{108} \zeta_{2}+\frac{41}{60} \zeta_{2}^{2}\right)+\varepsilon^{2}\left(\frac{3788165}{46656}-\frac{121}{30} \zeta_{5}-\frac{935}{324} \zeta_{3}\right. \\
& \left.\left.+\frac{22937}{1296} \zeta_{2}-\frac{13}{18} \zeta_{2} \zeta_{3}+\frac{97}{180} \zeta_{2}^{2}\right)\right]+C_{F}^{2}\left[\frac{1}{\varepsilon^{4}}(32)+\frac{1}{\varepsilon^{3}}(-48)\right. \\
& +\frac{1}{\varepsilon^{2}}\left(98-8 \zeta_{2}\right)+\frac{1}{\varepsilon}\left(-\frac{309}{2}+\frac{128}{3} \zeta_{3}\right)+\left(\frac{5317}{24}-90 \zeta_{3}+\frac{41}{2} \zeta_{2}-13 \zeta_{2}^{2}\right) \\
& +\varepsilon\left(-\frac{28127}{96}+\frac{92}{5} \zeta_{5}+\frac{1327}{6} \zeta_{3}-\frac{1495}{24} \zeta_{2}-\frac{56}{3} \zeta_{2} \zeta_{3}+\frac{173}{6} \zeta_{2}^{2}\right) \\
& +\varepsilon^{2}\left(\frac{1244293}{3456}-\frac{311}{10} \zeta_{5}-\frac{34735}{72} \zeta_{3}+\frac{652}{9} \zeta_{3}^{2}+\frac{38543}{288} \zeta_{2}+\frac{193}{6} \zeta_{2} \zeta_{3}-\frac{10085}{144} \zeta_{2}^{2}\right. \\
& \left.\left.+\frac{223}{20} \zeta_{2}^{3}\right)\right]+C_{A} C_{F}\left[\frac{1}{\varepsilon^{3}}\left(\frac{44}{3}\right)+\frac{1}{\varepsilon^{2}}\left(-\frac{332}{9}+4 \zeta_{2}\right)+\frac{1}{\varepsilon}\left(\frac{4921}{54}-26 \zeta_{3}\right.\right. \\
& \left.+\frac{11}{3} \zeta_{2}\right)+\left(-\frac{120205}{648}+\frac{755}{9} \zeta_{3}-\frac{251}{9} \zeta_{2}+\frac{44}{5} \zeta_{2}^{2}\right)+\varepsilon\left(\frac{2562925}{7776}-\frac{51}{2} \zeta_{5}\right. \\
& \left.-\frac{5273}{27} \zeta_{3}+\frac{14761}{216} \zeta_{2}+\frac{89}{6} \zeta_{2} \zeta_{3}-\frac{3299}{120} \zeta_{2}^{2}\right)+\varepsilon^{2}\left(-\frac{50471413}{93312}+\frac{3971}{60} \zeta_{5}\right. \\
& \left.\left.+\frac{282817}{648} \zeta_{3}-\frac{569}{12} \zeta_{3}^{2}-\frac{351733}{2592} \zeta_{2}-\frac{1069}{36} \zeta_{2} \zeta_{3}+\frac{7481}{120} \zeta_{2}^{2}-\frac{809}{280} \zeta_{2}^{3}\right)\right] .
\end{aligned}
$$


Open Access. This article is distributed under the terms of the Creative Commons Attribution License (CC-BY 4.0), which permits any use, distribution and reproduction in any medium, provided the original author(s) and source are credited.

\section{References}

[1] N. Arkani-Hamed, S. Dimopoulos and G. Dvali, The hierarchy problem and new dimensions at a millimeter, Phys. Lett. B 429 (1998) 263 [hep-ph/9803315] [INSPIRE].

[2] I. Antoniadis, N. Arkani-Hamed, S. Dimopoulos and G. Dvali, New dimensions at a millimeter to a Fermi and superstrings at a TeV, Phys. Lett. B 436 (1998) 257 [hep-ph/9804398] [INSPIRE].

[3] N. Arkani-Hamed, S. Dimopoulos and G. Dvali, Phenomenology, astrophysics and cosmology of theories with submillimeter dimensions and TeV scale quantum gravity, Phys. Rev. D 59 (1999) 086004 [hep-ph/9807344] [INSPIRE].

[4] L. Randall and R. Sundrum, A large mass hierarchy from a small extra dimension, Phys. Rev. Lett. 83 (1999) 3370 [hep-ph/9905221] [INSPIRE].

[5] ATLAS collaboration, Search for contact interactions and large extra dimensions in dilepton events from pp collisions at $\sqrt{s}=7 \mathrm{TeV}$ with the ATLAS detector, Phys. Rev. D 87 (2013) 015010 [arXiv:1211.1150] [InSPIRE].

[6] ATLAS collaboration, Search for Extra Dimensions in diphoton events using proton-proton collisions recorded at $\sqrt{s}=7 \mathrm{TeV}$ with the ATLAS detector at the LHC,

New J. Phys. 15 (2013) 043007 [arXiv:1210.8389] [InSPIRE].

[7] ATLAS collaboration, Search for Extra Dimensions using diphoton events in $7 \mathrm{TeV}$ proton-proton collisions with the ATLAS detector, Phys. Lett. B 710 (2012) 538 [arXiv: 1112.2194] [INSPIRE].

[8] CMS collaboration, Search for signatures of extra dimensions in the diphoton mass spectrum at the Large Hadron Collider, Phys. Rev. Lett. 108 (2012) 111801 [arXiv:1112.0688] [INSPIRE].

[9] CMS collaboration, Search for large extra dimensions in dimuon and dielectron events in $p p$ collisions at $\sqrt{s}=7 \mathrm{TeV}$, Phys. Lett. B 711 (2012) 15 [arXiv:1202.3827] [INSPIRE].

[10] P. Mathews, V. Ravindran, K. Sridhar and W. van Neerven, Next-to-leading order QCD corrections to the Drell-Yan cross section in models of TeV-scale gravity,

Nucl. Phys. B 713 (2005) 333 [hep-ph/0411018] [INSPIRE].

[11] P. Mathews and V. Ravindran, Angular distribution of Drell-Yan process at hadron colliders to NLO-QCD in models of TeV scale gravity, Nucl. Phys. B 753 (2006) 1 [hep-ph/0507250] [INSPIRE].

[12] M. Kumar, P. Mathews and V. Ravindran, PDF and scale uncertainties of various DY distributions in ADD and RS models at hadron colliders, Eur. Phys. J. C 49 (2007) 599 [hep-ph/0604135] [INSPIRE].

[13] M. Kumar, P. Mathews, V. Ravindran and A. Tripathi, Diphoton signals in theories with large extra dimensions to NLO QCD at hadron colliders, Phys. Lett. B 672 (2009) 45 [arXiv:0811.1670] [INSPIRE]. 
[14] M. Kumar, P. Mathews, V. Ravindran and A. Tripathi, Direct photon pair production at the LHC to order $\alpha_{s}$ in TeV scale gravity models, Nucl. Phys. B 818 (2009) 28 [arXiv:0902 .4894] [INSPIRE].

[15] N. Agarwal, V. Ravindran, V. Tiwari and A. Tripathi, Z boson pair production at the LHC to $O\left(\alpha_{s}\right)$ in TeV scale gravity models, Nucl. Phys. B 830 (2010) 248 [arXiv:0909.2651] [INSPIRE].

[16] N. Agarwal, V. Ravindran, V.K. Tiwari and A. Tripathi, $W^{+} W^{-}$production in Large extra dimension model at next-to-leading order in $Q C D$ at the $L H C$, Phys. Rev. D 82 (2010) 036001 [arXiv:1003.5450] [InSPIRE].

[17] N. Agarwal, V. Ravindran, V.K. Tiwari and A. Tripathi, Next-to-leading order QCD corrections to $W^{+} W^{-}$production at the LHC in Randall Sundrum model, Phys. Lett. B 690 (2010) 390 [arXiv: 1003.5445] [inSPIRE].

[18] R. Frederix et al., Diphoton production in the ADD model to NLO+parton shower accuracy at the LHC, JHEP 12 (2012) 102 [arXiv:1209.6527] [INSPIRE].

[19] R. Frederix, M. Mandal, P. Mathews, V. Ravindran and S. Seth, Drell-Yan, ZZ, W+Wproduction in SM 83 ADD model to NLO+PS accuracy at the LHC, arXiv:1307.7013 [INSPIRE].

[20] P. Nogueira, Automatic Feynman graph generation, J. Comput. Phys. 105 (1993) 279 [INSPIRE].

[21] J. Kuipers, T. Ueda, J. Vermaseren and J. Vollinga, FORM version 4.0, Comput. Phys. Commun. 184 (2013) 1453 [arXiv:1203.6543] [INSPIRE].

[22] R.J. Gonsalves, Dimensionally regularized two loop on-shell quark form-factor, Phys. Rev. D 28 (1983) 1542 [inSPIRE].

[23] G. Kramer and B. Lampe, Two Jet Cross-Section in $e^{+} e^{-}$Annihilation, Z. Phys. C 34 (1987) 497 [Erratum ibid. C 42 (1989) 504] [INSPIRE].

[24] T. Matsuura and W. van Neerven, Second Order Logarithmic Corrections to the Drell-Yan Cross-section, Z. Phys. C 38 (1988) 623 [INSPIRE].

[25] T. Matsuura, S. van der Marck and W. van Neerven, The Calculation of the Second Order Soft and Virtual Contributions to the Drell-Yan Cross-Section, Nucl. Phys. B 319 (1989) 570 [InSPIRE].

[26] R.V. Harlander, Virtual corrections to $g g \rightarrow H$ to two loops in the heavy top limit, Phys. Lett. B 492 (2000) 74 [hep-ph/0007289] [INSPIRE].

[27] C. Anastasiou and K. Melnikov, Higgs boson production at hadron colliders in NNLO QCD, Nucl. Phys. B 646 (2002) 220 [hep-ph/0207004] [INSPIRE].

[28] V. Ravindran, J. Smith and W.L. van Neerven, NNLO corrections to the total cross-section for Higgs boson production in hadron hadron collisions, Nucl. Phys. B 665 (2003) 325 [hep-ph/0302135] [INSPIRE].

[29] V. Ravindran, J. Smith and W. van Neerven, Two-loop corrections to Higgs boson production, Nucl. Phys. B 704 (2005) 332 [hep-ph/0408315] [INSPIRE].

[30] F. Tkachov, A Theorem on Analytical Calculability of Four Loop Renormalization Group Functions, Phys. Lett. B 100 (1981) 65 [INSPIRE]. 
[31] K. Chetyrkin and F. Tkachov, Integration by Parts: The Algorithm to Calculate $\beta$-functions in 4 Loops, Nucl. Phys. B 192 (1981) 159 [InSPIRE].

[32] W. van Neerven, Dimensional Regularization of Mass and Infrared Singularities in Two Loop On-shell Vertex Functions, Nucl. Phys. B 268 (1986) 453 [inSPIRE].

[33] R. Cutkosky, Singularities and discontinuities of Feynman amplitudes, J. Math. Phys. 1 (1960) 429 [inSPIRE].

[34] P. Baikov and V.A. Smirnov, Equivalence of recurrence relations for Feynman integrals with the same total number of external and loop momenta, Phys. Lett. B 477 (2000) 367 [hep-ph/0001192] [INSPIRE].

[35] T. Gehrmann, T. Huber and D. Maître, Two-loop quark and gluon form-factors in dimensional regularisation, Phys. Lett. B 622 (2005) 295 [hep-ph/0507061] [INSPIRE].

[36] T. Gehrmann and E. Remiddi, Differential equations for two loop four point functions, Nucl. Phys. B 580 (2000) 485 [hep-ph/9912329] [INSPIRE].

[37] V.A. Smirnov, Evaluating Feynman Integrals, Springer Tracts of Modern Physics Heidelberg, (2004).

[38] S. Laporta, High precision calculation of multiloop Feynman integrals by difference equations, Int. J. Mod. Phys. A 15 (2000) 5087 [hep-ph/0102033] [INSPIRE].

[39] C. Anastasiou and A. Lazopoulos, Automatic integral reduction for higher order perturbative calculations, JHEP 07 (2004) 046 [hep-ph/0404258] [INSPIRE].

[40] C. Studerus, Reduze-Feynman Integral Reduction in $\mathrm{C}++$, Comput. Phys. Commun. 181 (2010) 1293 [arXiv:0912.2546] [INSPIRE].

[41] A. von Manteuffel and C. Studerus, Reduze 2 - Distributed Feynman Integral Reduction, arXiv: 1201.4330 [INSPIRE].

[42] R. Lee, Presenting LiteRed: a tool for the Loop InTEgrals REDuction, arXiv:1212.2685 [INSPIRE].

[43] V. Sudakov, Vertex parts at very high-energies in quantum electrodynamics, Sov. Phys. JETP 3 (1956) 65 [INSPIRE].

[44] A.H. Mueller, On the Asymptotic Behavior of the Sudakov Form-factor, Phys. Rev. D 20 (1979) 2037 [INSPIRE].

[45] J.C. Collins, Algorithm to Compute Corrections to the Sudakov Form-factor, Phys. Rev. D 22 (1980) 1478 [InSPIRE].

[46] A. Sen, Asymptotic Behavior of the Sudakov Form-Factor in QCD, Phys. Rev. D 24 (1981) 3281 [INSPIRE].

[47] J.C. Collins, Sudakov form factors, in Perturbative QCD, Advanced Series on Directions in High Energy Physics: Vol. 5, A.H. Mueller ed., World Scientific, Singapore, (1989) ANL-HEP-PR-84-36.

[48] S. Moch and A. Vogt, Higher-order soft corrections to lepton pair and Higgs boson production, Phys. Lett. B 631 (2005) 48 [hep-ph/0508265] [INSPIRE].

[49] E. Laenen and L. Magnea, Threshold resummation for electroweak annihilation from DIS data, Phys. Lett. B 632 (2006) 270 [hep-ph/0508284] [INSPIRE]. 
[50] A. Idilbi, X.-d. Ji, J.-P. Ma and F. Yuan, Threshold resummation for Higgs production in effective field theory, Phys. Rev. D 73 (2006) 077501 [hep-ph/0509294] [INSPIRE].

[51] V. Ravindran, On Sudakov and soft resummations in QCD, Nucl. Phys. B 746 (2006) 58 [hep-ph/0512249] [INSPIRE].

[52] J. Kodaira and L. Trentadue, Summing Soft Emission in QCD, Phys. Lett. B 112 (1982) 66 [INSPIRE].

[53] S. Moch, J. Vermaseren and A. Vogt, The three loop splitting functions in QCD: The Nonsinglet case, Nucl. Phys. B 688 (2004) 101 [hep-ph/0403192] [INSPIRE].

[54] S. Catani, The singular behavior of QCD amplitudes at two loop order, Phys. Lett. B 427 (1998) 161 [hep-ph/9802439] [INSPIRE].

[55] G.F. Sterman and M.E. Tejeda-Yeomans, Multiloop amplitudes and resummation, Phys. Lett. B 552 (2003) 48 [hep-ph/0210130] [INSPIRE].

[56] T. Becher and M. Neubert, Infrared singularities of scattering amplitudes in perturbative QCD, Phys. Rev. Lett. 102 (2009) 162001 [arXiv:0901.0722] [INSPIRE].

[57] E. Gardi and L. Magnea, Factorization constraints for soft anomalous dimensions in QCD scattering amplitudes, JHEP 03 (2009) 079 [arXiv:0901.1091] [INSPIRE].

[58] C. Anastasiou, E.N. Glover, C. Oleari and M. Tejeda-Yeomans, Two loop QCD corrections to massless quark gluon scattering, Nucl. Phys. B 605 (2001) 486 [hep-ph/0101304] [INSPIRE].

[59] C. Anastasiou, E.N. Glover, C. Oleari and M. Tejeda-Yeomans, Two loop QCD corrections to massless identical quark scattering, Nucl. Phys. B 601 (2001) 341 [hep-ph/0011094] [INSPIRE].

[60] C. Anastasiou, E.N. Glover, C. Oleari and M. Tejeda-Yeomans, Two-loop QCD corrections to the scattering of massless distinct quarks, Nucl. Phys. B 601 (2001) 318 [hep-ph/0010212] [INSPIRE].

[61] E.N. Glover, C. Oleari and M. Tejeda-Yeomans, Two loop QCD corrections to gluon-gluon scattering, Nucl. Phys. B 605 (2001) 467 [hep-ph/0102201] [INSPIRE].

[62] P. Artoisenet et al., A framework for Higgs characterisation, JHEP 11 (2013) 043 [arXiv: 1306.6464] [INSPIRE].

[63] M. Kumar, P. Mathews, A. Pankov, N. Paver, V. Ravindran and A.V. Tsytrinov, Spin-analysis of s-channel diphoton resonances at the LHC, Phys. Rev. D 84 (2011) 115008 [arXiv:1108.3764] [INSPIRE].

[64] D. de Florian, M. Mahakhud, P. Mathews, J. Mazzitelli and V. Ravindran, Next-to-Next-to-Leading Order QCD Corrections in Models of TeV-Scale Gravity, arXiv: 1312.7173 [INSPIRE]. 